\title{
Regimes pluviais, estação chuvosa e probabilidade de ocorrência de veranicos no Estado do Ceará
}

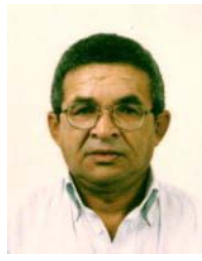

Francisco de A. S. e Silva ${ }^{1} \&$ Tantravahi V. R. Rao ${ }^{2}$

1 DEAg/CCT/UFCG. Av. Aprígio Veloso 882, Bodocongó, CEP 58109-970, Campina Grande, PB. Fone: (83) $331-3709$. E-mail: chaty@uol.com.br (Foto)

2 DCA/CCT/UFCG. Fone: (83) 310-1031: E-mail: ramana@dca.ufpb.br

Protocolo $119-6 / 8 / 2001$

\begin{abstract}
Resumo: Um método de regionalização pluvial proposto foi aplicado a dados pluviais mensais de 175 postos do Estado do Ceará e identificou três grupos pluvialmente homogêneos: grupo 1 (105 postos) predominantemente semi-árido, grupo 2 (56 postos) e grupo 3 (14 postos). Compararam-se esses grupos com as sete regiões climáticas reconhecidas pela Fundação Cearense de Meteorologia e Recursos Hídricos (FUNCEME) e constatou-se que o método foi capaz de identificar as regiões lbiapaba e Cariri, bem como a área composta pelas regiões Centro-Leste e Centro-Oeste e a área formada pelas regiões Litoral Norte e Litoral Sul. Identificou-se também, a estação chuvosa (EC) em cada posto do Estado do Ceará, mediante a utilização do método de Frère \& Popov (1979), e constatou-se que em $143(81,71 \%)$ dos 175 postos a EC ocorre de janeiro a maio e que em $69(65,71 \%)$ dos 105 postos do grupo 1, ela ocorre de fevereiro a maio. Verificou-se a probabilidade de ocorrência de veranicos nos três decêndios $(1$ a 10,11 a 20 e 21 ao último dia do mês) dos meses de janeiro a junho nos 105 postos do grupo 1 . Observou-se, ainda, probabilidades grupais médias de 51,39 e $25 \%$, respectivamente no primeiro, segundo e terceiro decêndio de janeiro; 20,18 e $21 \%$ em fevereiro; 10,7 e $6 \%$ em março; 9, 10 e $11 \%$ em abril; 25, 25 e $28 \%$ em maio e de 38 , 43 e $51 \%$ em junho.
\end{abstract}

Palavras-chave: grupos homogêneos, regionalização, precipitação pluvial, semi-árido

\section{Precipitation regimes, rainy season and the probability of occurrence of dry spells in the State of Ceará, Brazil}

\begin{abstract}
A regionalization method based on precipitation analysis proposed here is applied to the monthly precipitation data of 175 localities in Ceará State. It resulted in the identification of three homogeneous precipitation groups. Group 1 being predominantly semi-arid. Such groups were compared with the seven climatic regions recognized by 'Fundação Cearense de Meteorologia e Recursos Hídricos' (FUNCEME) and it was found that the method is capable of identifying the Ibiapaba and Cariri regions, as well as the area formed by the East Central and West Central regions and the area formed by the coastal regions of North and South. The rainy season for all the localities of the Ceará State was identified utilizing Frère \& Popov (1979) method. It was found that in 143 $(81.71 \%)$ of the 175 localities, the rainy season occurs from January to May and in $69(65.71 \%)$ of the 105 localities of Group1, the rainy season occurs from February to May. Finally, the daily precipitation data were used to verify the probability of occurrence of the dry spells in each of the three ten-day periods (days 1 to 10,11 to 20 and 21 to the last day of the month) for the months of January to June and for all the 105 locations of the Group 1. It was observed that the mean probability of having a dry spell in the first, second and third 10 day-periods of the month of January is 51, 39 and 25\%, respectively; in February it is 20, 18 and 21\%; in March 10, 7 and 6\%; in April 9, 10 and 11\%; in May 25, 25 and 28\%; and in June 38, 43 and 51\%.
\end{abstract}

Key words: homogeneous groups, regionalization, rainfall, semi-arid

\section{INTRODUÇÃO}

O Estado do Ceará, cuja superfície é de 146.817 km², está situado entre as latitudes 2 e $8^{\circ} \mathrm{S}$ e as longitudes 37 e $42^{\circ} \mathrm{W}$. Seu relevo tem altitude variada que, excetuando-se as elevações localizadas, cresce de norte para sul e de leste para oeste, sendo os postos pluviais Camocim ( $5 \mathrm{~m}$ ) e Mulungu (1050 m), de menor e maior altitudes, respectivamente. Em cerca de $75 \%$ da sua superfície ocorrem totais anuais menores que $1000 \mathrm{~mm}$. A grande variabilidade interanual e espacial da precipitação pluvial nessa região é fato constatado por pesquisadores, como Uvo \& Berndtsson (1996). A acentuada variabilidade interanual das chuvas no semi-árido do Nordeste brasileiro pode exceder os $40 \%$. O semi-árido nordestino ocupa grande parte da superfície 
do Estado do Ceará e em algumas localidades ocorrem totais anuais de chuva menores que $500 \mathrm{~mm}$. Essa adversidade climática se constitui num grande problema socioeconômico, visto que precipitações pluviais controlam a produção das culturas nos trópicos sazonalmente secos (Sansigolo, 1996).

No semi-árido cearense, concentrado nas regiões CentroLeste e Centro-Oeste, assim como em toda a área onde chove menos de $1000 \mathrm{~mm}$ anuais, além de anos secos e dos baixos totais pluviais anuais, a distribuição intra-anual da chuva também é adversa, já que concentra mais de $70 \%$ do total em apenas quatro meses. Nessa época o solo supostamente apresenta umidade adequada ao crescimento e ao desenvolvimento das principais culturas tradicionais da região como algodão, feijão e milho. Além dos eventuais anos secos e da curta duração da estação chuvosa (EC), a região ainda tem outra adversidade climática no tocante à exploração agrícola que é a ocorrência de períodos multidiários com pouquíssima ou nenhuma chuva durante a EC, conhecidos como veranicos. Eles se constituem num sério problema porque, quando vão além de aproximadamente dez dias, durante os estádios fenológicos de floração e de formação da produção causam frustração parcial ou total de safra. Esse tipo de evento é conhecido como "seca verde". Os veranicos também são muito prejudiciais quando ocorrem no período da emergência das plantas.

Os efeitos dos anos secos, a curta duração da EC e os dos veranicos poderão ser minimizados mediante a utilização de variedades de cultivos de ciclo curto e tolerantes à seca e, também, pela adoção de irrigação suplementar, dependendo do tipo de solo e da disponibilidade e qualidade da água. No planejamento de irrigação suplementar, em nível regional, é necessário saber-se a probabilidade de ocorrência de veranicos. Ademais, a regionalização pluvial e as informações sobre o início da EC podem ser de grande importância para programas de liberação de crédito rural e de insumos agrícolas.

O objetivo deste trabalho foi a identificação de grupos pluvialmente homogêneos e da estação chuvosa no Estado do Ceará e, ainda, verificar a probabilidade de ocorrência de veranicos nos decêndios da EC em localidades do semi-árido cearense.

\section{MATERIAL E MÉTODOS}

Foram utilizados dados diários e mensais de precipitação pluvial de 175 postos do Estado do Ceará, todos com mais de 30 anos de dados cujos períodos variam entre 1910 e 1985. Esses dados foram obtidos no Departamento de Ciências Atmosféricas, do Centro de Ciências e Tecnologia da Universidade Federal de Campina Grande (UFCG).

As temperaturas médias mensais do ar, utilizadas no cálculo da evapotranspiração potencial mensal, foram estimadas em função das coordenadas geográficas de cada posto (localidade) conforme o modelo proposto por Cavalcanti \& Silva (1994):

$$
\begin{aligned}
\mathrm{T} & =\mathrm{A}_{0}+\mathrm{A}_{1} \lambda+\mathrm{A}_{2} \phi+\mathrm{A}_{3} \mathrm{~h}+\mathrm{A}_{4} \lambda^{2} \\
& +\mathrm{A}_{5} \phi^{2}+\mathrm{A}_{6} \mathrm{~h}^{2}+\mathrm{A}_{7} \lambda \phi+\mathrm{A}_{9} \lambda \mathrm{h}+\mathrm{A}_{9} \phi \mathrm{h}
\end{aligned}
$$

em que $\lambda$ é a longitude, em graus; $\phi$ é a latitude, em graus e h é a altitude em metros. Os coeficientes $A_{0}, \ldots, A_{9}$ comuns aos Estados do Ceará, Rio Grande do Norte, Paraíba e Pernambuco, foram obtidos por Cavalcanti \& Silva (1994), para os doze meses do ano.

O método de regionalização proposto baseia-se no coeficiente de assimetria (a3m) das doze médias pluviais mensais (MM) e na média anual (MA), de cada posto, e consiste no seguinte:

\section{Cálculo de a3m para cada posto pluvial (localidade)}

Com base na definição do coeficiente de assimetria de Pearson, constante em Spiegel (1977), calcula-se:

$$
\mathrm{a} 3 \mathrm{~m}=\mathrm{m}_{3} /\left(\mathrm{m}_{2}\right)^{3 / 2}
$$

em que $m_{2}$ e $m_{3}$ são os momentos centrados na média. Como os valores de a3m são pequenos e podem ser negativos ou positivos, calcula-se a variável A3 para que se tenha uma melhor adequação do plano MA x a3m, sendo A3 definida conforme a seguinte expressão:

$$
\mathrm{A} 3=(\mathrm{a} 3 \mathrm{~m}+\mathrm{c}) \mathrm{x} 1000
$$

em que $\mathrm{c}=($ menor a $3 \mathrm{~m}$ negativo $) \times(-1)$ ou $\mathrm{c}=0$ (se não houver a3m negativo).

A constante "c" visa tornar todos os valores de A3 positivos, quando existem valores de “a $3 \mathrm{~m}$ ” negativos e o fator multiplicador, "1000", visa a utilização da mesma escala em ambos os eixos.

\section{Identificação dos grupos}

Identificam-se os grupos em razão das áreas do plano MA x A3 que apresentarem maior densidade de pontos. A delimitação dos grupos é feita com base no número de postos em cada 25 unidades de escala, do menor para o maior valor do eixo, de forma que os pontos de separação dos grupos estejam situados em intervalos de 25 unidades de escala com o menor número de postos possível. Esse critério é aplicado ao eixo A3 e, depois, ao eixo MA. Assim, primeiro se separam grupos com relação a A3, e depois, dentro de cada grupo de A3, separam-se grupos com relação a MA. Não havendo mais de um grupo com relação a A3, separam-se os grupos apenas com relação a MA.

Para verificação da validade estatística dos grupos identificados pelo método de regionalização, os valores de MA, de $\mathrm{A} 3$ e da distância euclidiana $\mathrm{D}(\mathrm{MA}, \mathrm{A} 3)$, relativa à origem, foram comparados da seguinte forma: grupo1 x grupo2, grupo1 x grupo3 e grupo 2 x grupo3 (Tabela 1), utilizando-se o teste de Mann-Withney (Teste U) para comparação de duas amostras, através do software ASSISTAT (Silva, 1996).

A evapotranspiração potencial mensal média (ETP), em mm, foi estimada para 175 postos do Estado do Ceará, com base no método de Thornthwaite (1957):

$$
\mathrm{ETP}_{\mathrm{i}}=16 \times\left(10 \times \mathrm{T}_{\mathrm{i}} / \mathrm{I}\right)^{\mathrm{a}}
$$

em que:

i $\quad-1($ jan $), 2($ fev $), \ldots, 12(\mathrm{dez})$ 
$\mathrm{T}_{\mathrm{i}} \quad$ - Temperatura $\left({ }^{\circ} \mathrm{C}\right)$

$$
\begin{gathered}
\mathrm{I}=\mathrm{i}_{1}+\mathrm{i}_{2}+\ldots+\mathrm{i}_{12} \\
\mathrm{I}_{1}=\left(\mathrm{T}_{1} / 5\right)^{1.514}, \ldots, \mathrm{i}_{12}=\left(\mathrm{T}_{12} / 5\right)^{1,514}
\end{gathered}
$$

$\mathrm{a}=6,75 \times 10^{-7} \times \mathrm{I}^{3}-7,71 \times 10^{-5} \times \mathrm{I}^{2}+1,792 \times 10^{-2} \times \mathrm{I}+0,49239$

A identificação da EC baseou-se no método de Frère \& Popov (1979), o qual caracteriza a "estação de crescimento" como sendo os meses nos quais a precipitação mensal média $<\overline{\mathrm{P}}>$ é maior ou igual à metade da evapotranspiração potencial mensal média $<\overline{\mathrm{ETP}}>$, somando-se o número de dias após o último mês no qual P - ETP/2 $\geq 0$.

A identificação da probabilidade de ocorrência de veranicos decendiais nos meses de janeiro a junho foi realizada para 105 postos (localidades) de uma área predominantemente semiárida do Estado do Ceará. Os decêndios foram definidos como sendo os dias compreendidos entre 1 e 10, 11 e 20 e 21 até o último do mês, respectivamente, o primeiro, segundo e terceiro decêndio do mês considerado. Os procedimentos adotados nos cálculos são apresentados nos itens seguintes.

As 18 séries decendiais de janeiro a junho, dos 105 postos do grupo 1, utilizadas no estudo da probabilidade de ocorrência de veranicos não tiveram suas falhas preenchidas. Trabalhouse apenas com os dados diários de precipitação existentes. Assim sendo, o tratamento das séries decendiais limitou-se à aplicação do teste de Dixon (Koch \& Rego, 1985), para detecção de valores singulares máximos e à substituição desses valores pelos respectivos valores limite do teste.

A probabilidade de ocorrência de veranicos, $\mathrm{Pab}(0 \mathrm{~mm})$, de cada decêndio dos meses de janeiro a junho, para cada posto (localidade) do grupo 1 (105 postos), foi identificada como sendo a probabilidade empírica, Fab0, dos valores nulos (precipitação pluvial igual a $0 \mathrm{~mm}$ ), de acordo com o modelo de Kimbal, através da seguinte expressão:

$$
\mathrm{Pab} 0=\mathrm{M} /(\mathrm{N}+1)
$$

em que:

$$
\begin{array}{ll}
\mathrm{M} & -1,2, . ., \mathrm{N} \\
\mathrm{N} & \text { - número de valores }
\end{array}
$$

As séries decendiais foram ajustadas à distribuição Pearson III ou gama incompleta, pelo método de Kolmorgorov \& Smirnov (Hann, 1977) da seguinte forma:

1. Calcula-se:

$$
a_{i}=\left|F\left(x_{i}\right)-P\left(x_{i}\right)\right|
$$

em que:

$$
\begin{array}{ll}
\mathrm{i} & -1,2,3, \ldots, \mathrm{N} \\
\mathrm{N} & - \text { número de valores da série }
\end{array}
$$

$\mathrm{F}\left(\mathrm{x}_{\mathrm{i}}\right)$ - a probabilidade empírica. Em geral usa-se a de Kimbal

$\mathrm{P}\left(\mathrm{x}_{\mathrm{i}}\right)$ - a probabilidade segundo a lei de distribuição adotada

2. Identifica-se $\mathrm{a}_{\max }$, ou seja; o máximo valor de $\mathrm{a}_{\mathrm{i}}$.
3. Identifica-se $\mathrm{a}_{\mathrm{n}, \alpha}$, tabelado para $\alpha=5 \%, 10 \%$ etc.

4. Decisão do teste:

Se $\mathrm{a}_{\max }$ menor ou igual a $\mathrm{a}_{\mathrm{n}, \alpha}, \mathrm{H}_{0}$ não é rejeitada, ou seja: a série se ajusta à lei de distribuição adotada (para ajuste).

Para se ter não apenas a probabilidade de veranicos absolutos $(0 \mathrm{~mm})$, mas também a probabilidade de ocorrência de algumas precipitações pluviais decendiais insuficientes para a exploração agrícola calculou-se, também, para cada decêndio, a probabilidade (mista) de ocorrência de uma precipitação maior ou igual a $0 \mathrm{~mm}$ e menor ou igual a $5 \mathrm{~mm}, \mathrm{Pab}(0-5)$, com base em Thom (1951) utilizando-se a seguinte expressão:

$$
\operatorname{Pab}(0-5)=\mathrm{Fab} 0+(1-\mathrm{Fab} 0) \times \mathrm{Pab}(1-5)
$$

em que:

Pab(1-5) - a probabilidade de ocorrência de uma precipitação maior ou igual a $1 \mathrm{~mm}$ e menor ou igual a $5 \mathrm{~mm}$, dada pela distribuição Pearson III ou gama incompleta.

Determinou-se ainda, para cada decêndio, o total pluvial, em $\mathrm{mm}$, com $75 \%$ de probabilidade de ser superado (Tc75\%Pac).

\section{RESULTADOS E DISCUSSÃO}

O resultado da regionalização pluvial no Estado do Ceará é apresentado na Figura 1. Observa-se que o grupo 1 (105 postos) ocupa toda a parte central do Estado, abrangendo a área situada entre as latitudes 3 e $7,5^{\circ} \mathrm{S}$ e entre as longitudes 38 e $41^{\circ} \mathrm{W}$, caracterizada como semi-árida por Samani \& Hargreaves (1985). O grupo 2 (56 postos) é, basicamente, constituído pela faixa

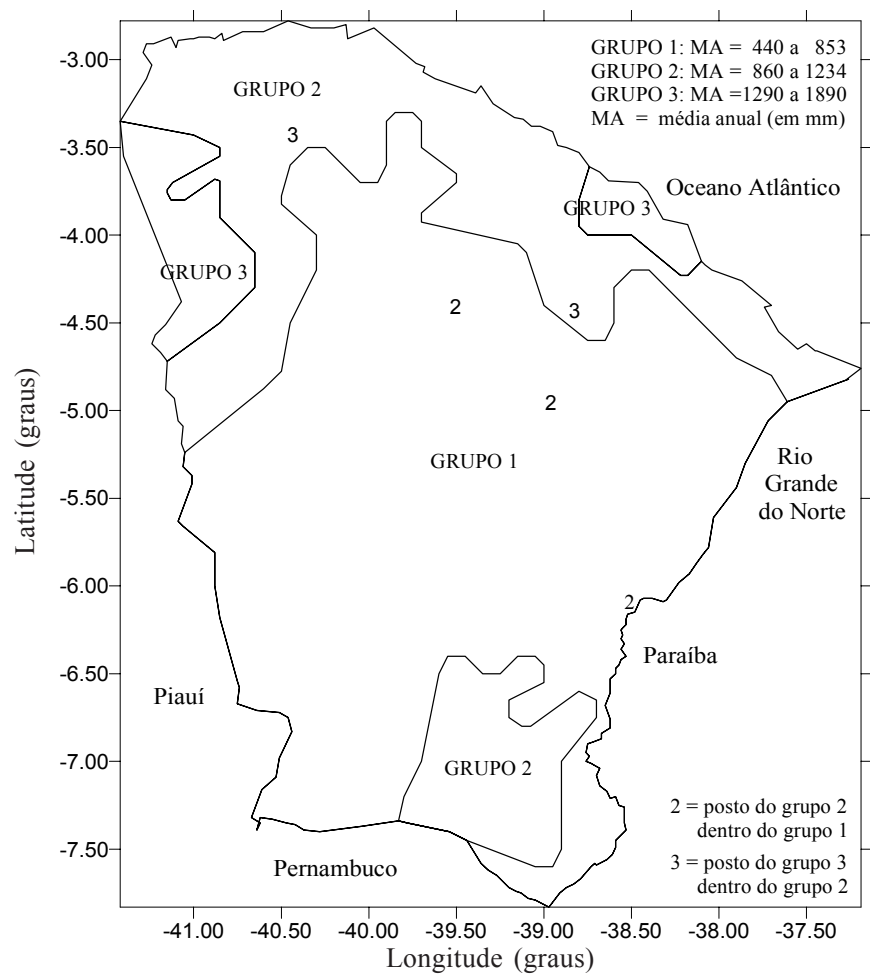

Figura 1. Delimitação dos grupos da regionalização da precipitação pluvial no Estado do Ceará 
litorânea e por um encrave dentro do grupo 1 , situado entre as latitudes 6,3 e $7,7^{\circ} \mathrm{S}$ e entre as longitudes 38,5 e $40^{\circ} \mathrm{W}$. O grupo 3 (14 postos) consiste em dois encraves de alta pluviosidade anual, sendo um no litoral, o qual abrange Fortaleza e adjacências, e outro a oeste, situado entre as latitudes 3 e $5^{\circ} \mathrm{S}$ e as longitudes 40,5 e $41,5^{\circ} \mathrm{W}$.

Comparando-se os três grupos obtidos com as sete regiões climáticas reconhecidas pela Fundação Cearense de Meteorologia e Recursos Hídricos - FUNCEME (Fig. 2), verificou-se correspondência entre o grupo 1 e as regiões Centro-Leste e Centro-Oeste. Quanto ao grupo 2, constatou-se uma correspondência entre a faixa litorânea e as regiões Litoral Norte e Litoral Sul. Em relação à média anual, essa faixa está coerente com as isoietas apresentadas por Uvo \& Berndtsson (1996). Também foi constatada correspondência entre o encrave (no grupo 1) e o Cariri, entretanto o mesmo não ocorre com parte do grupo 3, que abrange Fortaleza; para o outro encrave desse grupo (no grupo 2), constatou-se correspondência com a região Ibiapaba. Não foi constatada nenhuma correspondência com a região Baturité, mas, observou-se que essa região foi diferenciada com um posto isolado do grupo 3 , dentro do grupo 2, visto que essa área apresenta um microclima de serra, conforme observa-se nas isolinhas de altitude apresentadas na Figura 3.

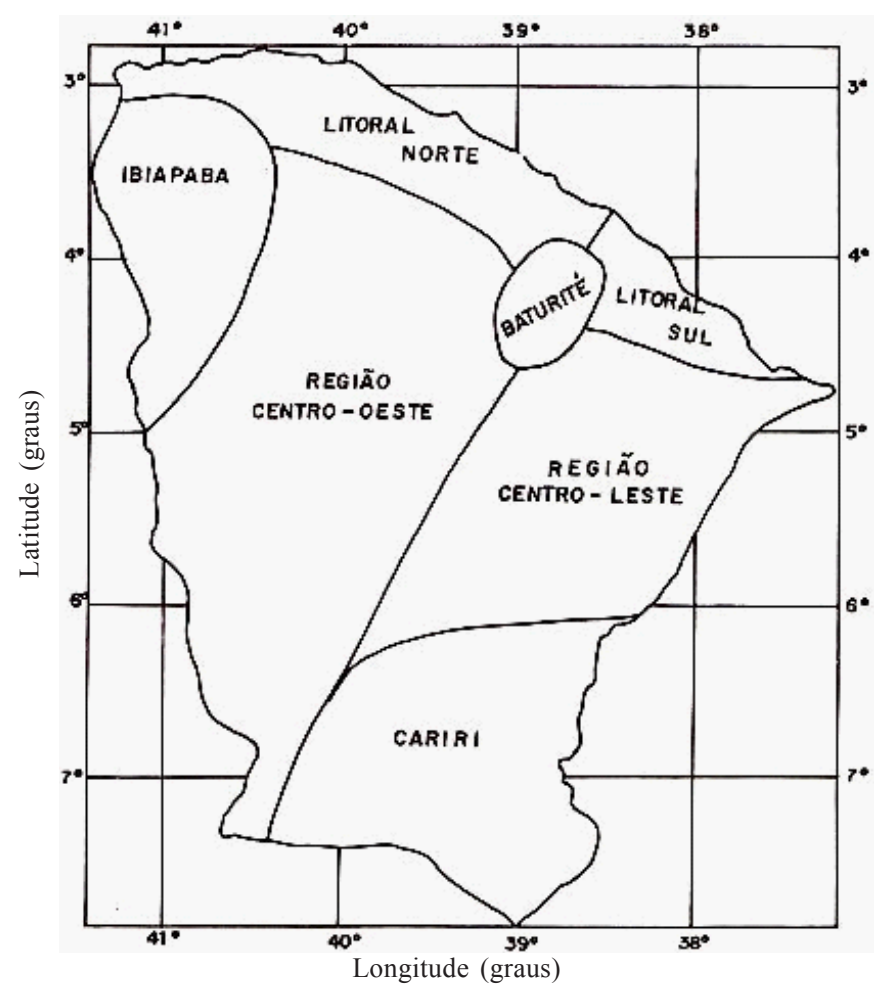

Figura 2. Regiões climáticas do Estado do Ceará reconhecidas pela FUNCEME (Fonte: FUNCEME, s/d)

Apesar do número de grupos ser diferente do número de regiões climáticas reconhecidas pela FUNCEME, o método de regionalização utilizado foi capaz de identificar diferenças regionais pluviais. Entretanto, não foi eficiente na identificação do número de grupos, devido provavelmente, de ter sido considerada apenas a altura de chuva e sua distribuição intraanual. As regiões da FUNCEME certamente não foram

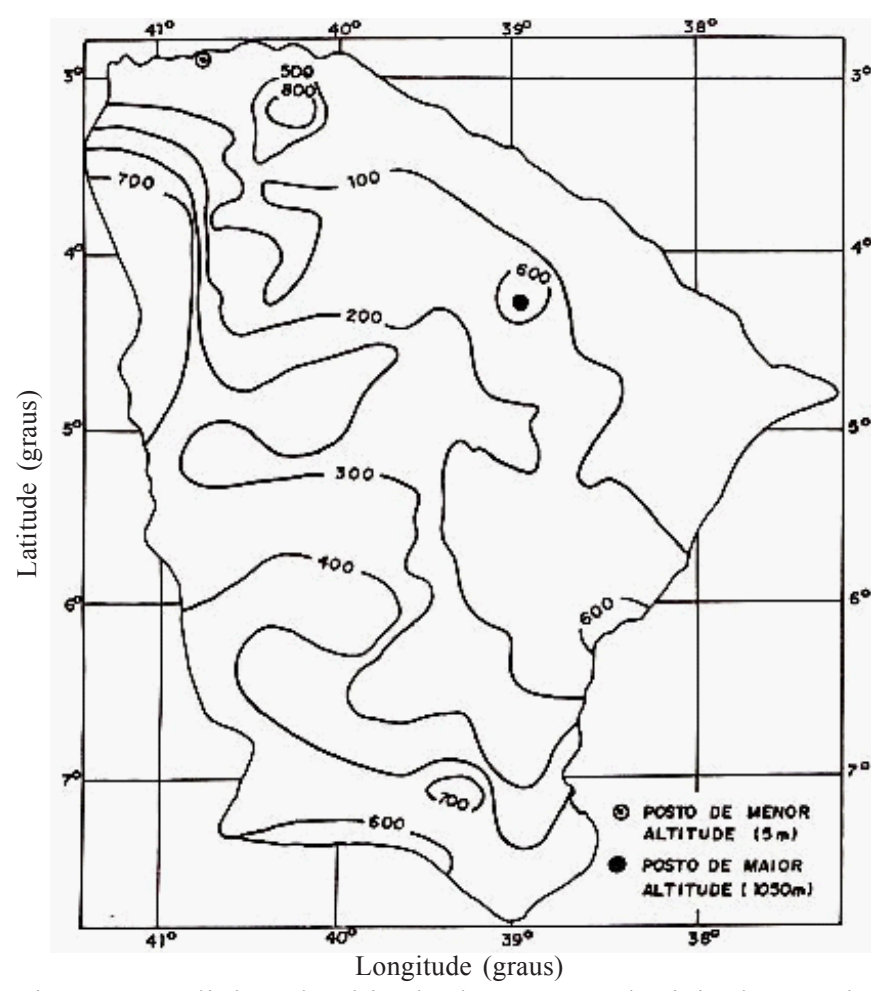

Figura 3. Isolinhas de altitude dos postos pluviais do Estado do Ceará

determinadas com base apenas nessas duas variáveis climáticas, por isso ressalta-se que isso não invalida os resultados obtidos diante dos objetivos deste trabalho. O número de grupos obtidos também difere daquele encontrado por Martins (1994), o qual obteve quatro grupos, utilizando-se da técnica hierárquica de "cluster analysis". Essa técnica, por si só, não define o número de grupos, o número deles (quatro) não deve ser tido como um valor correto desse parâmetro.

Por outro lado, Monteiro (1988) também identificou três regimes pluviais para o Estado do Ceará; contudo, não é oportuno comparar-se os três grupos com esses três regimes (grupos), porque Monteiro (1988) trabalhou apenas com 37 postos, porém a coincidência no número de grupos revela que o número deles obtido neste trabalho, não é aleatório considerando-se que Monteiro (1988) se baseou na comparação entre as curvas da precipitação média diária ao longo do ano, de cada posto, que é um critério de agrupamento inequívoco quando a regionalização é vista apenas com relação à altura pluvial.

A validade estatística dos grupos identificados no Estado do Ceará foi verificada através da aplicação do teste MannWithney (Teste U) para comparação de duas amostras, aos conjuntos de valores grupais da média anual (MA), da medida de assimetria (A3) e da distância euclidiana (DE) dos pontos (MA,A3) com relação à origem referentes aos grupos 1,2 e 3 . Os resultados da aplicação do referido teste são apresentados na Tabela 1.

Observou-se que apenas os conjuntos de valores da medida $\mathrm{A} 3$, do grupo 1 e do grupo 2 não são estatisticamente diferentes e como há conjuntos de médias anuais (MA) e/ou de coeficientes de assimetria das doze médias mensais (a3) ou, ainda conjuntos da medida (DE) baseada em MA e a3, referentes 
Tabela 1. Verificação da validade estatística dos grupos identificados feita através da aplicação do teste de MannWithney (Teste U) aos valores da MA, da A3 e da distância euclidiana (DE), dos pontos (MA,A3)

\begin{tabular}{cccc}
\hline Comparação & MA & A3 & DE \\
\hline Grupo 1 x Grupo 2 & $*$ & ns & $*$ \\
Grupo 1 x Grupo 3 & $*$ & $*$ & $*$ \\
Grupo 2 x Grupo 3 & $*$ & $*$ & $*$ \\
\hline
\end{tabular}

* - Significativo em nível de 0,05 de probabilidade

ns - não significativo

aos grupos identificados, estatisticamente diferentes entre si, isso revela que o método foi capaz de identificar grupos realmente diferentes entre si.

Com base na comparação da média pluvial com metade da evapotranspiração potencial, identificou-se a EC de cada posto (localidade), cuja distribuição espacial do seu início é observada na Figura 4. Apurou-se que em 83 dos 175 postos estudados do Estado, a EC se inicia em fevereiro, sendo que 78 destas pertencem ao grupo 1 , de acordo com a regionalização pluvial apresentada na Figura 1.

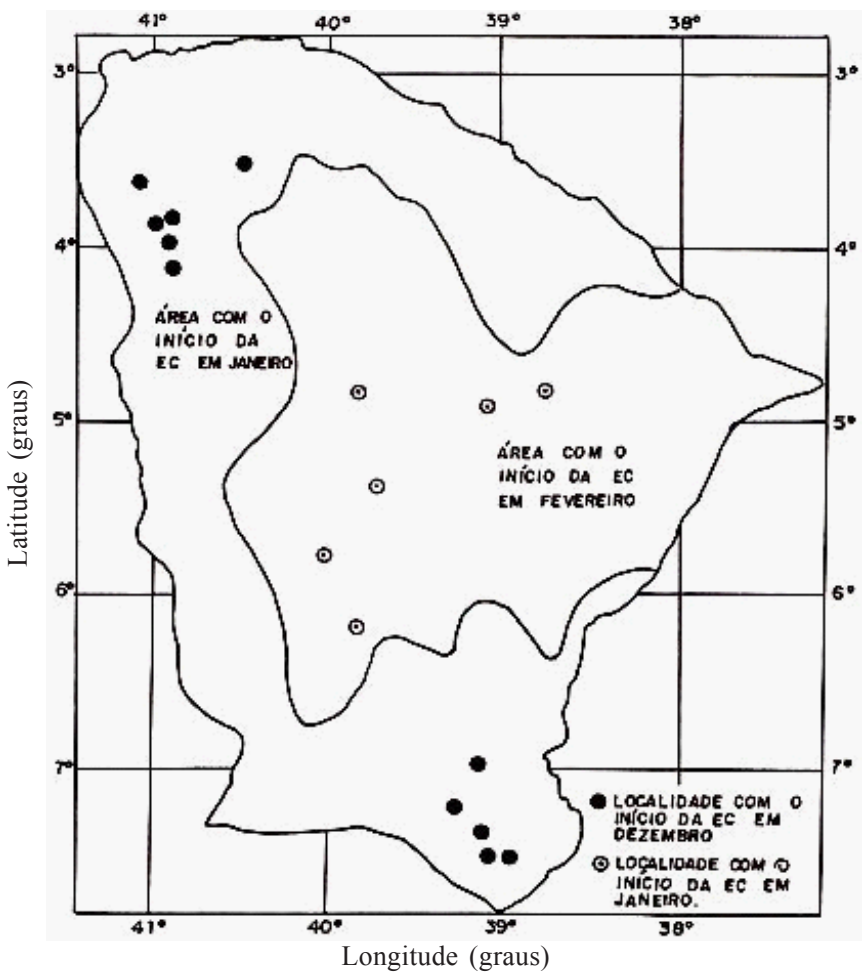

Figura 4. Distribuição espacial do início da estação chuvosa (EC) no Estado do Ceará

$\mathrm{Na}$ grande maioria dos postos a EC é de apenas quatro meses, ou seja, grande parte do total pluvial anual médio se concentra nos meses de fevereiro, março, abril e maio. Resultados semelhantes foram obtidos por Aldaz (1971), o qual observou que o trimestre mais chuvoso, na maior parte do Estado do Ceará, ocorre entre os meses de fevereiro e abril e na área Centro-Norte de março a maio.

As isolinhas da probabilidade média de ocorrência de veranicos, a probabilidade média de ocorrência de totais pluviais decendiais menores ou iguais a $5 \mathrm{~mm}$ e a média de totais pluviais decendiais esperados a $75 \%$ de probabilidade, dos 18 decêndios de janeiro a junho nos postos do grupo 1, estão apresentadas, respectivamente, nas Figuras 5, 6 e 7. De acordo com a Figura 5 constata-se que a probabilidade média de ocorrência de veranicos é de $30 \%$ em grande parte do grupo 1 , e que as menores probabilidades médias de ocorrência de veranicos, nesse grupo, estão em torno de $17 \%$. Esses resultados assim como as probabilidades médias de ocorrência de totais pluviais decendiais menores ou iguais a $5 \mathrm{~mm}$ (Fig. 6) e as médias dos totais pluviais decendiais esperados a $75 \%$ de probabilidade (Fig. 7) sugerem condições desfavoráveis à exploração agrícola em sistema de sequeiro.

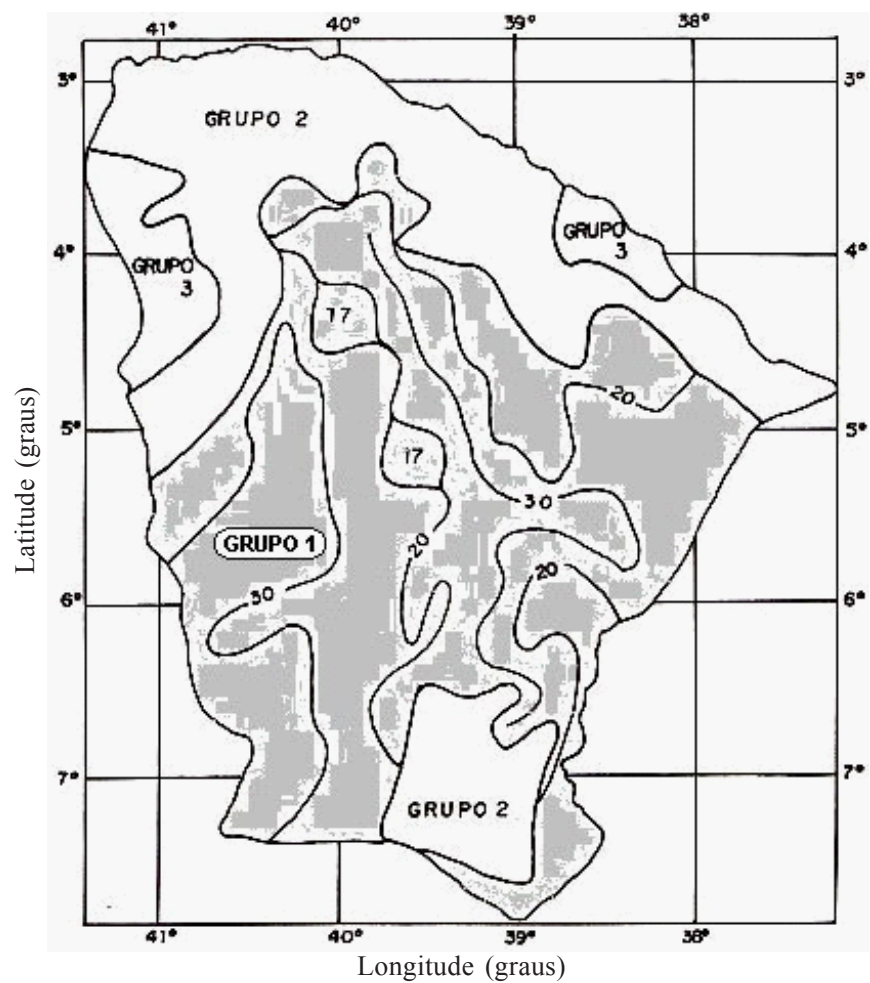

Figura 5. Isolinhas da probabilidade média de ocorrência de veranicos nos 18 decêndios de janeiro a junho no grupo 1

A média pluvial decendial em mm (Méd dec), o desviopadrão decendial (DPdec.) em mm, a probabilidade (\%) média de ocorrência de veranicos $(\mathrm{Pab}(0 \mathrm{~mm}))$, a probabilidade $(\%)$ média de ocorrência dos totais pluviais decendiais menores ou iguais a $5 \mathrm{~mm}(\mathrm{Pab}(0-5))$ e os totais pluviais decendiais, em mm, com $75 \%$ de probabilidade de serem superados (Tc $75 \%$ Pac), para os 105 postos do grupo 1, estão apresentados na Tabela 2. A probabilidade de ocorrência de precipitação decendial menor ou igual $5 \mathrm{~mm}$ é de aproximadamente $45 \%$ em fevereiro e $55 \%$ em maio, o que evidencia a semi-aridez da região do grupo 1. Outra constatação desfavorável para a exploração agrícola nessa região, é o fato que o total decendial da precipitação com $75 \%$ de probabilidade, tende para menos de $30 \mathrm{~mm}$, no terceiro decêndio e para menos de $21 \mathrm{~mm}$ nos segundo e terceiro decêndios de março, nos três decêndios de abril e para menos de $5 \mathrm{~mm}$ nos três decêndios de janeiro, fevereiro, maio e junho (Tabela 2). Esse resultado indica que a exploração agrícola em sistema de sequeiro na região do 


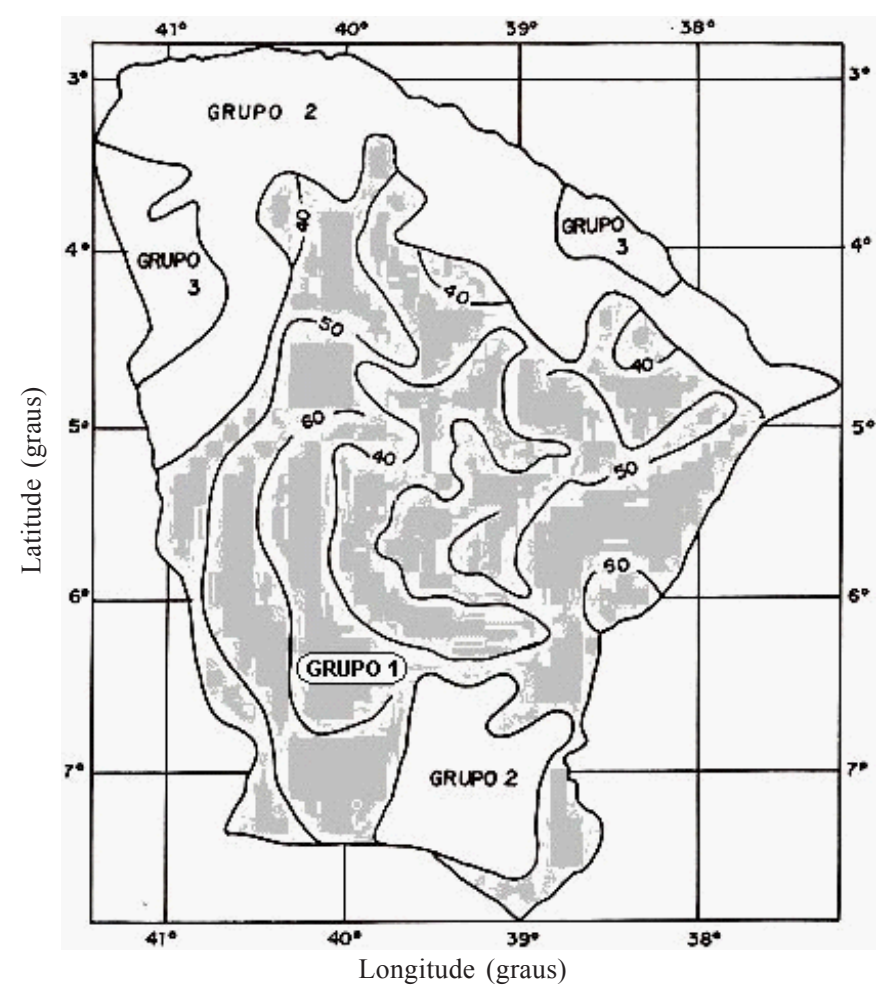

Figura 6. Isolinhas da probabilidade média de ocorrência de totais pluviais decendiais menores ou iguais a $5 \mathrm{~mm}$ nos 18 decêndios de janeiro a junho no grupo 1

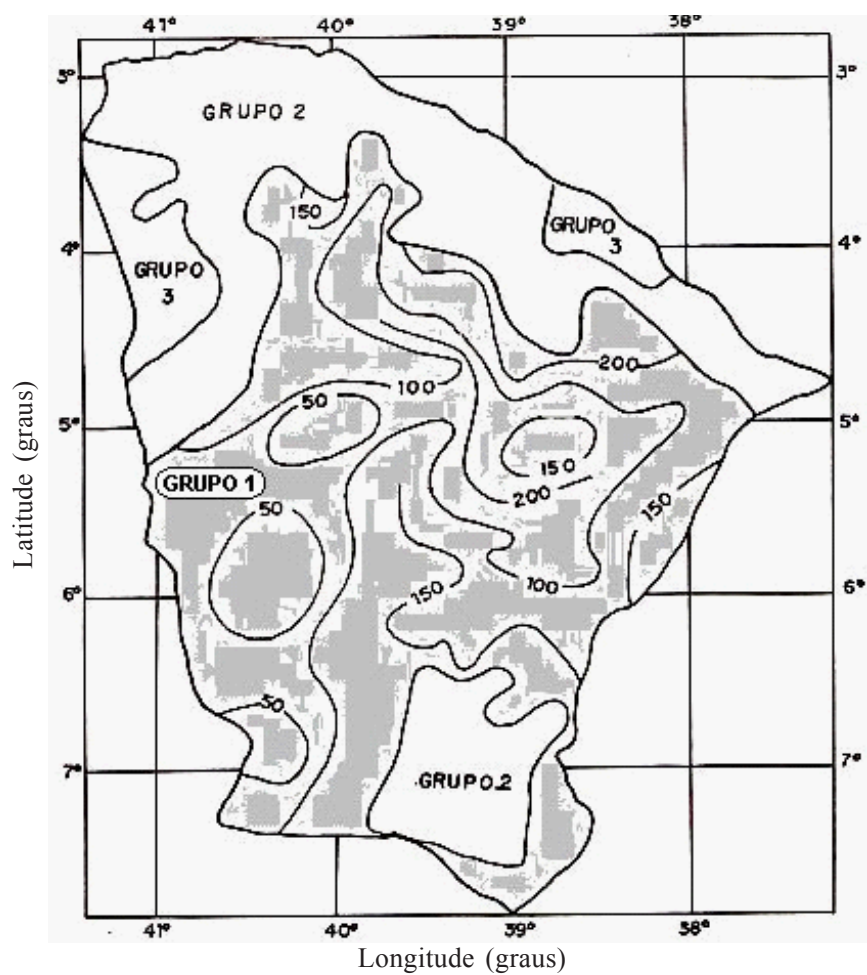

Figura 7. Isolinhas da média dos totais decendiais esperados a $75 \%$ de probabilidade nos 18 decêndios de janeiro a junho no grupo 1

Tabela 2. Valores da média pluvial decendial em milímetros (Méd dec), desvio-padrão decendial (D P dec), da probabilidade média de ocorrência de veranicos $(\mathrm{Pab}(0 \mathrm{~mm}))$, da probabilidade média de ocorrência de totais pluviais decendiais menores ou iguais a $5 \mathrm{~mm}(\mathrm{Pab}(0-5))$ e dos totais pluviais decendiais com $75 \%$ de probabilidade de serem superados (Tc75\%Pac) para os 105 postos do grupo $1^{*}$

\begin{tabular}{|c|c|c|c|c|c|c|c|c|c|c|c|c|c|c|c|c|c|c|}
\hline Meses & \multicolumn{3}{|c|}{ Janeiro } & \multicolumn{3}{|c|}{ Fevereiro } & \multicolumn{3}{|c|}{ Março } & \multicolumn{3}{|c|}{ Abril } & \multicolumn{3}{|c|}{ Maio } & \multicolumn{3}{|c|}{ Junho } \\
\hline Decêndio & $\mathrm{J} 1$ & $\mathrm{~J} 2$ & $\mathrm{~J} 3$ & F1 & F2 & F3 & M1 & M2 & M3 & $\mathrm{A} 1$ & A2 & A3 & M1 & M2 & M3 & J1 & $\mathrm{J} 2$ & $\mathrm{~J} 3$ \\
\hline Méd. dec. & 12 & 20 & 33 & 34 & 42 & 37 & 56 & 59 & 71 & 60 & 54 & 50 & 26 & 26 & 22 & 13 & 14 & 8 \\
\hline D.P.dec. & 21 & 30 & 39 & 39 & 44 & 39 & 52 & 52 & 56 & 51 & 50 & 45 & 31 & 31 & 26 & 18 & 21 & 12 \\
\hline $\mathrm{Pab}(0 \mathrm{~mm})$ & 51 & 39 & 25 & 20 & 18 & 21 & 10 & 7 & 6 & 9 & 10 & 11 & 25 & 25 & 28 & 38 & 43 & 51 \\
\hline $\operatorname{Pab}(0-5)$ & 78 & 73 & 52 & 45 & 43 & 43 & 30 & 23 & 18 & 24 & 29 & 26 & 55 & 55 & 56 & 67 & 74 & 79 \\
\hline $\mathrm{Tc} 75 \% \mathrm{Pac}$ & 0 & 0 & 1 & 3 & 4 & 2 & 15 & 19 & 29 & 20 & 15 & 15 & 1 & 1 & 2 & 0 & 0 & 0 \\
\hline
\end{tabular}

* Todas as probabilidades são espressas em percentagem

grupo 1 se constitui numa atividade de grande risco. Nesse sentido Pereira \& Medina (1983) sugeriram que um planejamento agrícola não deve basear-se em escores pluviais com probabilidade de ocorrência inferior a $75 \%$. Esses valores são típicos de regiões semi-áridas e coerentes com aqueles obtidos por Samani \& Hargreaves (1985), que baseados em totais pluviais mensais com esse mesmo nível de confiança classificaram a região central do Estado do Ceará como zona semiárida, e admitirem que há carência de irrigação suplementar nessa região.

A probabilidade de ocorrência de veranicos $(\mathrm{Pab}(0 \mathrm{~mm}))$ nos três decêndios dos meses de janeiro, fevereiro, março, abril, maio e junho nos 105 postos (localidades) do grupo 1, não apresentou nenhum valor que possa ser considerado singular, máximo ou mínimo. Em todos os postos essa probabilidade exibiu tendência lógica, ou seja, decrescendo cerca de $50 \%$ em primeiro decêndio de janeiro até menos de $10 \%$ em março, e crescendo de abril até o último decêndio de junho, quando volta a atingir cerca de $50 \%$ em junho. Esse comportamento da probabilidade de ocorrência de veranicos é inverso ao da média decendial e se reflete nos valores médios dos 105 postos (localidades) do grupo1, conforme se observa na Tabela 2 e na Figura 7.

\section{CONCLUSÕES}

1. O método de regionalização proposto, baseado na média pluvial anual e no coeficiente de assimetria das doze médias pluviais mensais, é capaz de identificar diferenças regionais pluviais no Estado do Ceará.

2. No Estado do Ceará existem três grupos pluvialmente homogêneos, sendo o maior deles predominantemente semiárido e ocupa toda a parte central do Estado.

3. A estação chuvosa na maior parte do Estado do Ceará ocorre entre os meses de janeiro e maio e na maior parte do semi-árido entre os meses de fevereiro e maio. 
4. A probabilidade de ocorrência de veranicos decendiais no semi-árido cearense é, respectivamente, de 51, 39 e 25\% em janeiro; de 20, 18 e 21\% em fevereiro; de 10, 7 e 6\% em março; de 9,10 e $11 \%$ em abril; de 25,25 e $28 \%$ em maio; e de 38,43 e $51 \%$ em junho.

5. A probabilidade média de ocorrência de precipitação total decendial menor ou igual $5 \mathrm{~mm}$, no semi-árido cearense é, respectivamente, de 78, 73 e $52 \%$ em janeiro; de 45,43 e $43 \%$ em fevereiro; de 30, 23 e 18\% em março; de 24, 29 e 26\% em abril; de 55,55 e $56 \%$ em maio; e de 67,74 e $79 \%$ em junho.

\section{LITERATURA CITADA}

Aldaz, L. A partial characterization of the rainfall regime of Brazil. Rio de Janeiro: DEMET/SUDENE/WMO, 1971, 104p.

Cavalcanti, E.P.; Silva, E.D.V. Estimativa da temperatura do ar em função das coordenadas locais. In: Congresso Brasileiro de Meteorologia, 7, Congresso Latino-Americano e Ibérico de Meteorologia, 2, 1994, Belo Horizonte. Anais... Belo Horizonte: SBMET, v.1, p.154-156, 1994.

Frère, M.; Popov, G.F. Agrometeorological crop monitoring and forecasting. Rome: FAO, 1979. p.31-40. Plant Production and Protection Paper, 17

Hann, C.T. Statistical methods in hydrology. Ames: Iowa University Press, 1977. p.176.

Koch, U.; Rêgo, J.C. Aplicação de testes não-paramétricos para verificar a homogeneidade de séries hidrometeorológicas. Revista Brasileira de Engenharia, Porto Alegre. v.3, n.1, p.47-76, 1985.

Martins, E.B. Distribuição espacial da chuva no Estado do Ceará - aplicação de técnica de classificação hierárquica. In: Congresso Brasileiro de Meteorologia, 8, Congresso LatinoAmericano e Ibérico de Meteorologia, 2, 1994, Belo Horizonte. Anais... Belo Horizonte: SBMET, 1994. v.2, p.336-337.
Monteiro, A.C.F. Uma delimitação dos regimes pluviais no Estado do Ceará. Campina Grande: CCT/UFPB, 1988. Relatório Técnico

Pereira, F.A.M.; Medina, B.F. Prognóstico de chuva para o município de Cruzeta-RN. In: Congresso Brasileiro de Engenharia Agrícola, 11, Brasília, 1981. Resumos... Brasília: Editerra, 1983.p.885-896.

Samani, Z.A.; Hargreaves, G.H. A crop water evaluation manual for Brazil. Logan: International Irrigation Center, 1985. $87 \mathrm{p}$.

Sansigolo, C.A. Variabilidade interanual da estação chuvosa no Estado de São Paulo. Revista Brasileira de Agrometeorologia, Santa Maria, v.4, n.1, p.101-105. 1996.

Silva, F. de A.S. e. The ASSISTAT Software: Statistical assistance. In: International Conference on Computers in Agriculture, 6, 1996, Cancun. Anais... Cancun: American Society of Agricultural Engineers, 1996. p.294-298.

Spiegel, M.R. Estatística: resumo da teoria, 875 problemas resolvidos, 619 problemas propostos. São Paulo: McGrawHill, 1977.580p

Thom, H.C.S. A frequency distribution for precipitation. Bulletin of the American Meteorological Society, Boston. v.32, n.10, p.397, 1951.

Thornthwaite, C.W. Instructions and tables for computing potencial evapotranspiration and the water balance. Publications in Climatology, New Jersey, v.10, n.3, p.185-311, 1957

Uvo, C.B.; Berndtsson, R. Regionalization and spatial properties of Ceará State rainfall in Northeast Brazil. Journal of Geophysical Research, Washington, v.101, p.4221-4233, 1996. 